\title{
HIV-1 evolution and drug resistance among patients receiving antiretroviral therapy in San Mateo county, California, 1997-2006
}

\author{
Sudeb Dalai ${ }^{1 *}$, Jonathan Dyal ${ }^{1}$, Keyan Salari ${ }^{1}$, Seble Kassaye ${ }^{1}$, Vivian Levy $^{2}$, Dennis Israelski ${ }^{2}$, David Katzenstein ${ }^{1}$ \\ From $16^{\text {th }}$ International Symposium on HIV and Emerging Infectious Diseases \\ Marseille, France. 24-26 March 2010
}

\section{Background}

In community public health antiretroviral therapy (ART) programs, HIV-1 viremia in patients has been reduced over the last decade through improved drug regimens, adherence, and genotypic testing to manage ART. However, a large portion (up to 25\%) of ART patients remain viremic with risk of disease progression and community-level transmission of drug resistance mutations. We hypothesized that HIV-1 viral evolutionary rate (vEvol) during incomplete suppression of HIV-1 RNA is associated with ART use, selection of drug resistance, and viral load.

\section{Methods}

HIV-1 reverse transcriptase (RT) and protease (Pr) sequences were obtained from 306 patients receiving ART at San Mateo Medical Center (1997-2006). From 150 paired sequences in 75 viremic patients, vEvol was determined using the TVM+I+G nucleotide substitution model in PAUP. Resistance mutations and genotypic susceptibilities to 19 ARV drugs were determined using the HIVseq algorithm (HIVDB.stanford.edu) and drug resistance mutations were correlated using hierarchical agglomerative clustering analysis. Statistical analysis was done in SAS v9.1.

\section{Results}

75 paired genotypes were a median of 10.9 months apart with median CD4 cells 260/cu mm and geometric mean RNA VL $3.94 \log$ copies/mL. ART was RTI-based (reverse-transcriptase inhibitor) in $38(51 \%)$ vs. PI-based (protease inhibitor) in 37 individuals (49\%), with similar rates of drug resistance mutations (89\%) in either drug class. Median vEvol was higher in those with PI- (but not RTI) associated drug resistance mutations ( $\mathrm{p}<0.05$, Wilcoxon rank-sum [WRS]). Higher vEvol was associated with reduced genotypic susceptibility scores to nRTI, nnRTI, and PI drugs ( $<<0.0001$, Spearman rank correlation). Hierarchical cluster analyses of all 306 sequences revealed many highly correlated drug resistance mutation pairs including T215Y/M41L in RT, V82/I54 in Pr, and M184V/L90M in RT/Pr, each being associated with higher vEvol ( $\mathrm{p}<0.05$, WRS).

\section{Discussion}

Repeated HIV-1 RNA genotyping in viremic patients showed significant drug resistance mutations in $89 \%$ and evolutionary changes in $\mathrm{RT} / \mathrm{Pr}$ in approximately $75 \%$. Viral evolution was significantly greater among those with higher HIV-1 VL, exposure to PI drugs and specific patterns of resistance mutations. The accumulation of drug resistance mutations in explicit patterns during ART treatment is an evolutionary response to drug selective pressure driven by virus replication.

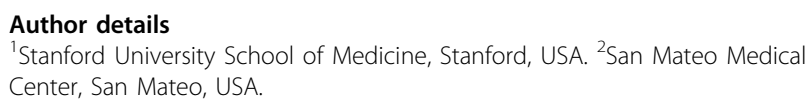

Published: 11 May 2010

\section{doi:10.1186/1742-4690-7-S1-P20}

Cite this article as: Dalai et al: HIV-1 evolution and drug resistance among patients receiving antiretroviral therapy in San Mateo county, California, 1997-2006. Retrovirology 2010 7(Suppl 1):P20.

* Correspondence: sdalai@stanford.edu

'Stanford University School of Medicine, Stanford, USA 